\title{
Controlled VLS Growth of Indium, Gallium and Tin Oxide Nanowires via Chemical Vapor Transport
}

\author{
M.C. Johnson ${ }^{1}$, S. Aloni ${ }^{2}$, D.E. McCready ${ }^{3}$, E.D. Bourret-Courchesne ${ }^{1, *}$ \\ ${ }^{1}$ Lawrence Berkeley National Laboratory, Materials Sciences Division, 1 Cyclotron Road, MS2R0200, \\ Berkeley, CA 94720-8197 \\ ${ }^{2}$ Lawrence Berkeley National Laboratory, Molecular Foundry, 1 Cyclotron Road, MS2R0200, Berkeley, \\ CA 94720-8197 and Department of Physics, University of California, Berkeley, CA 94720 \\ ${ }^{3}$ Environmental Molecular Sciences Laboratory, Pacific Northwest National Laboratory,, P.O. Box 999, \\ MS K8-93, Richland, WA 99352.
}

\begin{abstract}
We utilized a vapor-liquid-solid growth technique to synthesize indium oxide, gallium oxide, and tin oxide nanowires using chemical vapor transport with gold nanoparticles as the catalyst. Using identical growth parameters we were able to synthesize single crystal nanowires typically 40-100 nm diameter and more than 10-100 microns long. The products were characterized by means of XRD, SEM and HRTEM. All the wires were grown under the same growth conditions with growth rates inversely proportional to the source metal vapor pressure. Initial experiments show that different transparent oxide nanowires can be grown simultaneously on a single substrate with potential application for multi-component gas sensors.
\end{abstract}

Keywords: A. Oxides; B. Vapor deposition; C. Electron microscopy; C. X-ray diffraction; D. Crystal structure

Corresponding Author. Phone: 1-510-486-5553, Fax: 1-510-486-5530, E-mail address: EDBourret@1bl.gov 


\section{Introduction}

One-dimensional nanostructures, such as wires, rods, belts and tubes, have recently attracted much attention due to their novel properties and potential applications in nanoelectronics and nanophotonics ${ }^{1-6}$. Over the last decade, semiconductor 1D nanostructures such as carbon nanotubes, III-V, II-VI, Si-Ge, and oxides have been synthesized and studied for their nanoscale properties. Their reduced dimensionality leads to higher quality, defect free material with high surface to volume ratio. The latter is now utilized in the construction of gas sensing devices when the surface properties are modified by adsorption of gas which in turn affects the electronic properties of the wire. When the size of the nanowire is smaller then the exciton's Bohr radius in the bulk, the electronic properties are defined by the size due to quantum confinement effects. The metal oxides are extremely important technological materials for use in nanophotonic and nanoelectronic devices. Oxides such as $\mathrm{ZnO}^{7}, \mathrm{GeO}_{2}{ }^{8-9}, \mathrm{In}_{2} \mathrm{O}_{3}{ }^{10-12}, \mathrm{SnO}_{2}{ }^{13}, \mathrm{Ga}_{2} \mathrm{O}_{3}{ }^{14}$, etc. have been synthesized into nanowire and nanobelt structures and were successfully integrated into numerous optoelectronic applications and nanoscopic gas sensors ${ }^{15-18}$.

The first step in utilization of nanostructures is the development of simple, reproducible and controllable growth strategies for obtaining high quality and high purity materials. Many growth strategies have been employed to synthesize nanosized semiconducting oxides that have resulted in many different structures with various morphologies and properties. For instance, $\operatorname{In}_{2} \mathrm{O}_{3}$ nanowires have been grown using different vapor transport schemes utilizing various catalysts. Vapor-liquid-solid (VLS) mechanisms 
using catalytic Au thin films were utilized to obtain n-type $\operatorname{In}_{2} \mathrm{O}_{3}$ nanowires ${ }^{10}$. The $\mathrm{In}$ was delivered to the substrate via laser ablation of an InAs target which requires expensive and specific equipment and apparatuses for synthesis. Zhang, et al. used Ag catalyst and reduced $\operatorname{In}_{2} \mathrm{O}_{3}$ powders to obtain $\operatorname{In}_{2} \mathrm{O}_{3}$ nanowires ${ }^{11}$. The wires had a rough morphology and a wide size distribution (10 -100 nm). More difficult techniques involved the electrodeposition of In nanowires using templates of anodic alumina membranes (AAMs) which involves a multi-step process ${ }^{12}$. The In is then oxidized for as long as 12 hours. Although this method allows for dense arrays of nanowires, the products have rough morphologies and the synthesis involves many advanced processing steps and long annealing times. Tin oxide nanowires have been grown using selfcatalytic thermal evaporation ${ }^{21-23}$. These self-catalytic methods result in single crystal wires but with wide size distribution and various shapes. The self-catalytic process results in nanowires with rough morphology and leads to branching and dendritic growth ${ }^{22,23}$. High quality $\mathrm{SnO}_{2}$ wires have been obtained using electrochemical methods inside an alumina porous matrix ${ }^{24}$. Unfortunately this method takes a significant number of complex steps and involves long growth times. VLS techniques have also been reported for the synthesis of $\mathrm{Ga}_{2} \mathrm{O}_{3}$ nanowires ${ }^{14,25}$. Use of a thin layer of $\mathrm{Au}(25-100 \AA)$ catalyst on Si substrates while flowing Ar carrier gas over heated Ga metal has resulted in high purity, single crystal nanowires. This technique leads to nanowires having a diameter range of $\sim 80-350 \mathrm{~nm}^{14}$. Other VLS techniques use $\mathrm{GaN}$ as the Ga source while using In as the catalyst. This method produced single crystalline wires with a diameter range of $20-60 \mathrm{~nm}$. One drawback is that this method uses another Group III metal as a catalyst which has potential for impurity substitution during synthesis. Other more 
advanced techniques use carbothermal reduction techniques ${ }^{17}$ and metalorganic chemical vapor deposition $^{26}$. These methods have complex chemistries and result in varying shapes and crystal quality. As just described, there are many different approaches for synthesis of oxide nanowires resulting in a wide variety of size, shape, and morphology. These growth techniques vary more widely when comparing synthesis of different material systems such as indium oxide, gallium oxide and tin oxide. In this study we demonstrate that the growth of $\mathrm{In}_{2} \mathrm{O}_{3}, \mathrm{Ga}_{2} \mathrm{O}_{3}$ and $\mathrm{SnO}_{2}$ nanowires can be promoted by a simple, reproducible and mutually compatible strategy using identical growth parameters. Our synthesis implements a VLS growth mechanism using gold nanoparticles as the catalyst. Finally, we show results of initial experiments that lead to simultaneous synthesis of $\mathrm{In}_{2} \mathrm{O}_{3}$ and $\mathrm{SnO}_{2}$ nanowires on a single $\mathrm{Si}$ substrate.

\section{Experimental Section}

Substrates of $\mathrm{Si}(100)$ were cut into $10 \mathrm{~mm} \times 10 \mathrm{~mm}$ squares and prepared by degreasing via sonication in Trichloroethylene, Acetone, Methanol, and DI water for 15 minutes each. Then the sample was dried using ultrapure $\mathrm{N}_{2}$ and heated to approximately $110^{\circ} \mathrm{C}$. After being heated, a solution of $50 \mathrm{~nm}$ Au particles (Ted Pella, Inc.) with a mean diameter of $52.7 \mathrm{~nm}$ was dropped onto the substrate. The density of the Au particles varied depending on the amount of solution dropped onto the substrate. High purity $(6 \mathrm{~N})$ metal reactant species (In, Ga, or $\mathrm{Sn}$ ) were separately placed in a quartz boat and loaded into the center of a $25 \mathrm{~mm}$ fused quartz tube placed inside a horizontal tube furnace. Substrates were placed 2-5 $\mathrm{mm}$ downstream of the metal reactant species. The furnace 
was heated between $800-1000^{\circ} \mathrm{C}\left(60^{\circ} \mathrm{C} / \mathrm{min}\right)$ under various flowrates of $\mathrm{N}_{2}(100-1000$ sccm) which contained sufficient amounts of oxygen precursors for synthesis. The nitrogen gas was supplied from the burn-off of a liquid nitrogen tank. The growth times varied between 0.5 to 3 hours. Samples were cooled to room temperature under $\mathrm{N}_{2}$ before being unloaded from the reaction furnace. Crystal structure and phase were defined using Grazing Incidence X-Ray Diffraction (GIXRD). Morphology and composition were determined using SEM equipped with Energy Dispersive Spectroscopy (EDS). Growth direction, crystallinity, and defect morphology were investigated using TEM with Nanobeam Diffraction (NBD). The GIXRD apparatus was a Philips X'Pert MPD system equipped with a $\mathrm{Cu}$ source $(\lambda=1.5406 \AA)$ and parallel beam optics. The incident beam angle $(\Omega)$ was fixed at $2.5^{\circ}$, the scan range was $15.00^{\circ}-75.00^{\circ} 2 \Theta$, and the scan rate was $0.05^{\circ} / 45 \mathrm{~s}$. Data analysis was accomplished using the software program JADE (Materials Data Inc., Livermore, CA) and the Powder Diffraction File database (International Centre for Diffraction Data, Newtown Square, PA). SEM was performed using a FEI Sirion 75 microscope equipped with an EDAX system, and high resolution TEM was performed using a JEOL 200 operating at 200kV capable of NBD.

\section{Results and Characterization}

\section{A. Indium Oxide Nanowires}

Indium oxide nanowires were grown by placing high purity indium in the center of a tube furnace at $900^{\circ} \mathrm{C}$ and flowing $\mathrm{N}_{2}$ as the carrier gas. Figure 1a shows a low magnification SEM image where there is a high density of long, straight nanowires. Typical lengths for these nanowires were typically 2-10 microns (dependent on growth time) with a radius of 
$50-100 \mathrm{~nm}$ giving an average growth rate of $4 \mu \mathrm{m} / \mathrm{hr}$. A typical XRD spectrum is attached to Figure 1a where the diffracted peaks are indexed and indicate cubic phase material. The strong intensities of the $\operatorname{In}_{2} \mathrm{O}_{3}$ peaks and the absence of any indium metal peaks indicate that the $\operatorname{In}_{2} \mathrm{O}_{3}$ nanowires were of high purity, single-phase material. The lattice parameter was calculated to be $\mathrm{a}=1.0115 \mathrm{~nm}$ which is in good agreement with reported values $(\mathrm{a}=1.0118 \mathrm{~nm})$. The compositional purity of these nanowires was verified using EDS. A typical EDS spectrum is attached to Figure 1a which shows that the individual nanowires consist of only In an O. The Si signal was from the substrate material. A high magnification image is shown in Figure 1b. The nanowires have a very smooth morphology with a cubic cross-section. Compositional analysis at the tip of the nanowire (Figure $1 \mathrm{~b}$, inset) shows that it consists of $\mathrm{In}, \mathrm{O}$, and $\mathrm{Au}$. The presence of a $\mathrm{Au}$ nanoparticle at the tip indicates that these nanowires are grown via a VLS growth mechanism. HRTEM images (Figure 2) reveals that a randomly selected $\operatorname{In}_{2} \mathrm{O}_{3}$ nanowire is very straight with a uniform diameter of $65 \mathrm{~nm}$. The NBD pattern (inset, Figure 2b) confirms the XRD data that the nanowires are cubic $\mathrm{In}_{2} \mathrm{O}_{3}$ with a growth direction of $<100>$. The high resolution image shows defect-free material.

It is worth reporting that the growth of octahedral structures were found during some of the $\operatorname{In}_{2} \mathrm{O}_{3}$ nanowire synthesis runs although there was no change in growth conditions. Figure 1c shows a SEM image of some results that consist of Au tipped nanowires growing from large $\operatorname{In}_{2} \mathrm{O}_{3}$ octahedral bases. These octahedral structures were grown via a vapor-solid (VS) mechanism as reported by Hao, et $a l^{27}$ and will be discussed later. EDS spectra of these structures showed the base and nanowire to be $\operatorname{In}_{2} \mathrm{O}_{3}$ with the tip being 
Au. This was not a common growth result where most wires were grown perpendicular to the substrate surface without base formation.

\section{B. Gallium Oxide Nanowires}

To obtain $\mathrm{Ga}_{2} \mathrm{O}_{3}$ nanowires, the same growth conditions as those used for $\mathrm{In}_{2} \mathrm{O}_{3}$ nanowires were utilized except that high purity Ga metal was used instead of In. The SEM image of the resulting $\mathrm{Ga}_{2} \mathrm{O}_{3}$ nanowires is shown in Figure 3a. The nanowires have average lengths greater than $20 \mu \mathrm{m}$ with typical diameters between $40-50 \mathrm{~nm}$. The inset shows a high magnification image of a nanowire with a diameter of $40 \mathrm{~nm}$. The attached XRD spectrum reveals that the nanowires were monoclinic with lattice constants of $\mathrm{a}=$ $1.222 \mathrm{~nm}, \mathrm{~b}=0.3041 \mathrm{~nm}, \mathrm{c}=0.5808 \mathrm{~nm}$ and $\beta=103.8^{\circ}$. These values are in good agreement with reported bulk data $(\mathrm{a}=1.227 \mathrm{~nm}, \mathrm{~b}=0.3039 \mathrm{~nm}, \mathrm{c}=0.5808 \mathrm{~nm}$, and $\beta=$ $103.8^{\circ}$ ). The $\mathrm{Ga}_{2} \mathrm{O}_{3}$ nanowires had an average growth rate of $14 \mu \mathrm{m} / \mathrm{hr}$ which was 3 to 4 times greater than the $\operatorname{In}_{2} \mathrm{O}_{3}$ nanowires at the same growth conditions. Ga metal has a vapor pressure of $10^{-4}$ at $900^{\circ} \mathrm{C}$ which is 1.5 orders of magnitude lower than that of In metal at the same conditions. Thus the resulting increase in growth rate indicates that the growth is not limited by the metal vapor transport.

It is interesting to note that the $\mathrm{Ga}_{2} \mathrm{O}_{3}$ growth also contained structures consisting of triple junction wire nodes. Figure $3 \mathrm{~b}$ shows a SEM image of a junction growth for the $\mathrm{Ga}_{2} \mathrm{O}_{3}$ nanowires. These junctions could be due to nucleation on twins or to the presence of a small Au particle on the wire. These junctions have widths and diameters greater than 1 $\mu \mathrm{m}$ and therefore were too thick to perform TEM. 
Figure 3a shows the typical EDS spectrum for a $\mathrm{Ga}_{2} \mathrm{O}_{3}$ nanowire. The compositional make-up for the nanowires was $\mathrm{Ga}$ and $\mathrm{O}$ with no detection of any impurities above the detection limits with the exception of the Si substrate. EDS was also performed on the junction nodes of these wires with the same results. Figure 4a shows a TEM image of a single nanowire that is $40 \mathrm{um}$ long and $40 \mathrm{~nm}$ in diameter. The nanowire is extremely straight and smooth with a Au tip at the slightly tapered end as shown with the EDS spectrum in the inset. This indicates that the $\mathrm{Ga}_{2} \mathrm{O}_{3}$ nanowires were also grown via a VLS growth mechanism. Figure $4 \mathrm{~b}$ shows a defect-free, high resolution image of the nanowire with an interatomic spacing of $\mathrm{c}=5.6 \AA$. The inset shows a NBD pattern which confirms a single crystal wire with a growth direction of $<001>$.

\section{Tin Oxide Nanowires}

Tin oxide nanowires were also grown at the same growth conditions using high purity tin metal. Figure 5 shows the results for a typical growth run of bulk nanowires. XRD analysis shown in Figure 5a shows that tin oxide nanowires were grown with the cassiterite (tetragonal) crystallographic configuration with lattice constants measured to be $\mathrm{a}=0.4737 \mathrm{~nm}$ and $\mathrm{c}=0.3185 \mathrm{~nm}$ which is consistent with the bulk data $(\mathrm{a}=.04738$ $\mathrm{nm}, \mathrm{c}=0.3187 \mathrm{~nm})$. Figure 5a also shows the SEM and EDS results for the growth of tin oxide nanowires. The tin oxide nanowires are shown to be $50-75 \mathrm{~nm}$ in diameter with average lengths exceeding $50 \mu \mathrm{m}$. This corresponds to an average growth rate of approximately $20 \mu \mathrm{m} / \mathrm{hr}$. These wires are smooth with constant diameters for the length 
of the wires. The EDS analysis shows that the wires consist of Sn and O with no evidence of impurity detection.

The HRTEM verified that the morphology of the nanowires was extremely smooth and the diameter is constant along the length of these wires (Figure 5b). The insets show that the nanowires were single crystalline. NBD analysis verifies that the tin oxide nanowire has a growth direction of $<100>$ and the interatomic spacing was $4.7 \AA$ which is consistent for cassiterite $\mathrm{SnO}_{2}$. These data show the $\mathrm{SnO}_{2}$ nanowires to be high quality, defect-free material.

\section{Discussion}

Growth parameter conditions have been established to synthesize single crystal $\operatorname{In}_{2} \mathrm{O}_{3}$, $\mathrm{Ga}_{2} \mathrm{O}_{3}$, and $\mathrm{SnO}_{2}$ nanowires. Structural and morphological characterization showed the nanowires to be extremely high quality and defect free. These results show that all three types of wires to be extremely smooth which is not always consistent in the literature for oxide nanowires $^{11-13,26,27}$. Compositional analysis showed the tips to consist of gold nanoparticles saturated with metal and oxygen atoms which is consistent with a VLS growth mechanism. Table 1 gives a summary of physical and growth parameters for nanowires synthesis. The nanowire diameters are relatively constant where the diameter of the resulting wire is dependent on the size of nanoparticle catalyst used. The diameters for all three systems range between $40-100 \mathrm{~nm}$ which is consistent with the size of catalyst used for our synthesis (nominal - $50 \mathrm{~nm} \mathrm{Au} \mathrm{nanoparticles).} \mathrm{There} \mathrm{is} \mathrm{some} \mathrm{range}$ in diameter due to the aggregation of Au nanoparticles. 
The average Au catalyst particle was advertised as $52.7 \mathrm{~nm}$ as determined by the vendor. It was found that the average diameter size ranged from 50-100nm for $\operatorname{In}_{2} \mathrm{O}_{3}, 40-60 \mathrm{~nm}$ for $\mathrm{Ga}_{2} \mathrm{O}_{3}$, and 50-100 $\mathrm{nm}$ for $\mathrm{SnO}_{2}$. The larger diameter nanowires were determined to be due to catalyst particle aggregation. As the temperature was increased to $900^{\circ} \mathrm{C}$, surface mobility of the catalyst particles increased while the surfactant was being burned off. This allows the catalyst particles to agglomerate into larger size particles. Experiments were conducted to decrease the density of particles on the surface by diluting the catalyst solution with different diluents ( $\mathrm{DI}_{2} \mathrm{O}$, methanol, acetone) to different concentrations. This was done in the hope of decreasing the chance of particles aggregating at the higher temperatures. It turns out that larger diameter wires were still grown using the lowest particle concentrations (1:10 by volume) that still allow for acceptable amounts of nanowire synthesis. Other techniques are needed for controlled catalyst deposition which will have a more controlled effect on diameter size which is beyond the scope of this paper.

While the $\mathrm{In}_{2} \mathrm{O}_{3}$ synthesis produced mainly single crystal nanowires, Figure 1c shows the growth of two octahedron $\operatorname{In}_{2} \mathrm{O}_{3}$ structures forming the base of nanowires. The octahedron structures are large with sizes on the order of $1 \mu \mathrm{m}$. EDS and XRD analysis also shows that these structures consist of only cubic $\operatorname{In}_{2} \mathrm{O}_{3}$ without the presence of any catalyst material. Therefore it is believed that these structures are formed by a VS growth mechanism instead of the VLS growth mechanism that forms nanowires. Hao et al. report $\mathrm{In}_{2} \mathrm{O}_{3}$ octahedron CVD growth using In metal and Ar carrier gas on Si substrates ${ }^{27}$. They describe this growth as a vapor-solid mechanism and analyze the products in terms 
of surface energies resulting in different sizes and shapes. They also describe the synthesis of imperfect octahedrons due to long growth times ( $>2$ hours) and attribute this effect to structural defects such as oxygen vacancies, indium interstitials, oxygen interstitials, and oxygen antisite defects. Our results agree very well with their reported results. The shape of our octahedron bases are imperfect due to the long growth times used for growth of the nanowires. Also, it was determined that these octahedron structures were not due to a catalyst mediated growth mode verifying a VS growth mechanism.

We found that the growth rate of these nanowires increases in the order of $\operatorname{In}_{2} \mathrm{O}_{3}, \mathrm{Ga}_{2} \mathrm{O}_{3}$, and $\mathrm{SnO}_{2}$, respectively. This is inversely proportional to the vapor pressure of the respective reactant metal species at the growth temperature. As the vapor pressure of the metal reactant increases the growth rate of the nanowire decreases as depicted in Figure 6. This increased metal vapor pressure causes an increased suppression of the already limited oxygen content in the $\mathrm{N} 2$ carrier gas which, in turn, limits the transport of oxygen vapor to the growing catalyst interface. Therefore the limiting reactant in these growing nanowires is the small amount of residual oxygen which is supplied from the flowing nitrogen. One possible way to control and optimize the growth rate is to supply a diluted $\mathrm{O}_{2} /$ inert gas mixture. Our experiments show that large amounts of oxygen at high growth temperatures induce rapid oxidation of the reactant metal which results in an oxide coating. This, in turn, prevents the evaporation of the metal reactant source. 
The growth times for the synthesis of different oxide nanowires varied between 0.5 and 3 hours. The synthesis of all three oxide nanowires showed consistent kinetic data for all growth times. The increase in growth time only resulted in an increase in nanowire length with diameters remaining constant. This is further proof that the diameter of nanowire is dependent on catalyst particle size. More importantly, there were no changes in crystallinity or morphological properties with wire length (i.e. growth time). Experiments were conducted a minimum of five times and showed a consistent reproducibility in growth and structural properties. This simple and reproducible synthesis technique of high quality nanowires implies an attractive method for future development of multicomponent oxide nanowire devices.

Initial experiments were performed for the growth of multiple types of nanowires on a single substrate. By simultaneously flowing In and Sn metal vapor at the same growth conditions used for single type wire growth $\left(900^{\circ} \mathrm{C}, 500 \mathrm{sccm} \mathrm{N} \mathrm{N}_{2}, 50 \mathrm{~nm}\right.$ Au catalyst $)$ a dual growth mode was achieved. Formation of $\operatorname{In}_{2} \mathrm{O}_{3}$ and $\mathrm{SnO}_{2}$ nanowires on a single substrate was confirmed by XRD. Diffraction data showed peaks for both cubic $\operatorname{In}_{2} \mathrm{O}_{3}$ and tetragonal $\mathrm{SnO}_{2}$ wires which verifies they exist separately while there was no diffraction peaks for ternary indium tin oxide nanowires. These initial experiments show the potential for simultaneously growing oxide nanowires on a single substrate at a single growth condition. Research is being performed on the development of controlled growth of multiple wire types as well as the ternary compounds. 


\section{Conclusion}

A simple VLS growth strategy has been developed to synthesize high quality $\operatorname{In}_{2} \mathrm{O}_{3}$, $\mathrm{Ga}_{2} \mathrm{O}_{3}$, and $\mathrm{SnO}_{2}$ nanowires. All three oxide nanowire systems can be grown under identical conditions using a gold catalyst material. Structural and morphological characterizations have shown that the nanowire are single crystal with extremely smooth morphologies. The diameters range from 40-100nm with lengths from several microns to over $50 \mu \mathrm{m}$. It has been shown that the growth rate of the different nanowires is inversely proportional to the vapor pressure of the respective metal reactant species. This is due to the displacement of oxygen precursor as the metal reactant vapor pressure increases. These nanowires can be grown simultaneously on a single substrate which has potential uses for multicomponent gas phase detection. It also implies that ternary or doped materials can be grown using simple CVD methods.

\section{Acknowledgement}

This work was supported by the Director, Office of Science, Office of Basic Energy Sciences, Division of Materials Science and Engineering, of the U.S. Department of Energy under Contract No. DE-AC02-05CH11231. The research described in this paper was performed [in part] in the Environmental Molecular Sciences Laboratory, a national scientific user facility sponsored by the US Department of Energy's Office of Biological and Environmental Research and located at Pacific Northwest National Laboratory in Richland, WA. 


\section{References}

(1) S. Iijima, Nature (London) 354 (1991) 56.

(2) J. Kong, C. Zhou, A. Morpurgo, H.T. Soh, C.F. Quate, C. Marcus, H. Dai, Appl. Phys. A 69 (1999) 305.

(3) J.T. Hu, T.W. Odom, C.M. Lieber, Acc. Chem. Res. 32 (1999) 435.

(4) Y. Cui, J. Lauhon, M.S. Gudiksen, J.Wang, C.M. Lieber, Appl. Phys. Lett. 78 (2001) 2214.

(5) X. Duan, C.M. Lieber, J. Am. Chem. Soc. 122 (2000) 188.

(6) Y. Xia, P. Yang, Y. Sun, Y. Wu, B. Mayers, B.Gates, Y. Yin, F. Kim, H. Yan, Adv. Mater. 15 (2003) 353.

(7) M.H. Huang, Y. Wu, H. Feick, N. Tran, E. Weber, P. Yang, Adv. Mater. 13 (2001) 113.

(8) X.C. Wu, W.H. Song, M.H. Pu, B. Zhao, Y.P. Sun, J.J. Du, Chem. Phys. Lett. 349 (2001) 210 .

(9) Z.G. Bai, D.P. Yu, H.Z. Zhang, Y. Ding, Y.P. Wang, X.Z. Gai, Q.L. Hang, G.C. Xiong, S.Q. Feng, Chem. Phys. Lett. 303 (1999) 311.

(10) C. Li, D. Zhang, S. Han, X. Liu, T. Tang, C. Zhou, Adv. Mater. 15 (2003) 143.

(11) J. Zhang, X. Qing, F. Jiang, Z. Dai, Chem. Phys. Lett. 371 (2003) 311.

(12) M.J. Zheng, L.D. Zhang, G.H. Li, X.Y. Zhang, X.F. Wang, Appl. Phys, Lett. 79 (2001) 839 .

(13) Z.R. Dai, J.L. Gole, J.D. Stout, Z.L. Wang, J. Phys. Chem. B 106 (2002) 1274.

(14) P. Guha, S. Chakrabarti, S. Chaudhuri, Physica E 23 (2004) 81. 
(15) C. Li, D. Zhang, X. Liu, S. Han, T. Tang, J. Han, C. Zhou, Appl. Phys. Lett. 82 (2002) 1613.

(16) D. Zhang, C. Li, X. Liu, S. Han, T. Tang, C. Zhou, Appl. Phys. Lett. 83 (2003) 1845.

(17) X.C. Wu, W.H. Song, W.D. Huang, M.H. Pu, B. Zhao, Y.P. Sun, J.J. Du, Chem.

Phys. Lett. 328 (2000) 5.

(18) M. Law, H. Kind, F. Kim, B. Messer, P. Yang, Angew. Chem. Int. Ed. 41 (2002) 2405.

(19) H.J. Chun, Y.S. Choi, S.Y. Bae, H.C. Choi, J. Park, Appl. Phys. Lett. 85 (2004) 461.

(20) B. Lei, C. Li, D. Zhang, T. Tang, C. Zhou, Appl. Phys. A 79 (2004) 439.

(21) N.S. Ramgir, I.S. Mulla, K.P. Vijayamohanan, J. Phys. Chem. B 108 (2004) 14815.

(22) Y. Chen, X. Cui, K. Zhang, D. Pan, S. Zhang, B. Wang, J.G. Hou, Chem. Phys. Lett. $369(2003) 16$.

(23) Y.J. Ma, F. Zhou, L. Lu, Z. Zhang, Solid State Comm. 130 (2004) 313.

(24) Y. Zhang, A. Kolmakov, S. Chreitien, H. Metiu, M. Moskovits, NanoLett. 4 (2004) 403.

(25) J. Zhang, F. Jiang, Chem. Phys. 289 (2003) 243.

(26) H.W. Kim, N.H. Kim, Appl. Surf. Sci. 233 (2004) 294.

(27) Y. Hao, G. Meng, C. Ye, L. Zhang, Crystal Growth \& Design, 5(2005)1617. 


\section{Table 1}

Growth parameters and physical properties of $\mathrm{In}_{2} \mathrm{O}_{3}, \mathrm{Ga}_{2} \mathrm{O}_{3}$, and $\mathrm{SnO}_{2}$ nanowires grown via a VLS mechanism using metal vapor transport for a 30 minute growth time.

\begin{tabular}{|c|c|c|c|}
\hline Parameter & $\mathrm{In}_{2} \mathrm{O}_{3}$ & $\mathrm{Ga}_{2} \mathrm{O}_{3}$ & $\mathrm{SnO}_{2}$ \\
\hline Growth Temperature $\left({ }^{\circ} \mathrm{C}\right)$ & $\overline{900}$ & $\overline{900}$ & $\overline{900}$ \\
\hline Catalyst & 50nm Au & 50nm Au & 50nm Au \\
\hline $\mathrm{N}_{2}$ Flow Rate $(\mathrm{sccm})$ & 500 & 500 & 500 \\
\hline Diameter (nm) & $50-100$ & $40-60$ & $50-100$ \\
\hline Average Growth Rate $(\mu \mathrm{m} / \mathrm{hr})$ & 4 & 14 & 20 \\
\hline Metal Vapor Pressure (Torr) & $3 \times 10^{-3}$ & $1 \times 10^{-4}$ & $3 \times 10^{-5}$ \\
\hline Crystalline phase & Cubic & Monoclinic & Tetragonal \\
\hline
\end{tabular}




\section{Figure Captions}

Figure 1 - $\mathrm{SEM}$ analysis for $\mathrm{In}_{2} \mathrm{O}_{3}$ nanowires grown at $900^{\circ} \mathrm{C}$ using Au catalyst. (a) Low magnification image of bulk nanowires. Inset shows XRD spectrum with cubic phase material. EDS inset shows wires to consist of In and $O$ only. Si peak is from the substrate. (b) High magnification image of wires showing gold tips. Inset shows EDS spectrum taken at the tip verifying presence of Au. (c) Low magnification image of nanowires grown from large $\mathrm{In}_{2} \mathrm{O}_{3}$ octahedron base. These structures are formed due to a $\mathrm{V}-\mathrm{S}$ growth mechanism as reported by Hao, et al. ${ }^{27}$.

Figure 2 - TEM analysis for $\operatorname{In}_{2} \mathrm{O}_{3}$ nanowires grown at $900^{\circ} \mathrm{C}$ using Au catalyst. (a) Low magnification image of single nanowire with diameter of $65 \mathrm{~nm}$ and smooth surface morphology. (b) High magnification image of defect-free nanowire. Insets show an atomically resolved image and a NBD spectrum indicating a growth direction of $<100>$.

Figure 3 - $\mathrm{SEM}$ analysis for $\mathrm{Ga}_{2} \mathrm{O}_{3}$ nanowire grown at $900^{\circ} \mathrm{C}$ using Au catalyst. (a) Low magnification image showing bulk wires with lengths exceeding $20 \mu \mathrm{m}$. Inset is high magnification image of single wire with diameter of $40 \mathrm{~nm}$. Side insets include XRD and EDS spectrums showing single phase monoclinic material consisting of only Ga and $\mathrm{O}$. (b) High magnification image showing junction node of $\mathrm{Ga}_{2} \mathrm{O}_{3}$ nanowire.

Figure 4 - TEM analysis for $\mathrm{Ga}_{2} \mathrm{O}_{3}$ nanowires grown at $900^{\circ} \mathrm{C}$ using Au catalyst. (a) Low magnification image of single nanowire with a diameter of $40 \mathrm{~nm}$ with a metal tip. The inset shows an EDS spectrum of the tip of the nanowire verifying that the tip consists of gold catalyst. (b) High magnification image of defect free nanowire. Insets show an atomically resolved image and a NBD spectrum indicating a growth direction of $<002>$.

Figure 5 - SEM and TEM analysis for $\mathrm{SnO}_{2}$ nanowires grown at $900^{\circ} \mathrm{C}$ using $\mathrm{Au}$ catalyst. (a) Low magnification SEM image of long, smooth nanowires. Inset shows a smooth nanowire with a diameter of $100 \mathrm{~nm}$. Side insets include XRD and EDS spectrums showing single phase tetragonal material consisting of only Sn and O. (b) High Magnification TEM image of defect free nanowire. Insets show an atomically resolved image and a NBD spectrum indicating a growth direction of $<100>$.

Figure 6 - Comparison of growth rate and metal vapor pressure for all three metal oxide nanowires. 
Figure 1
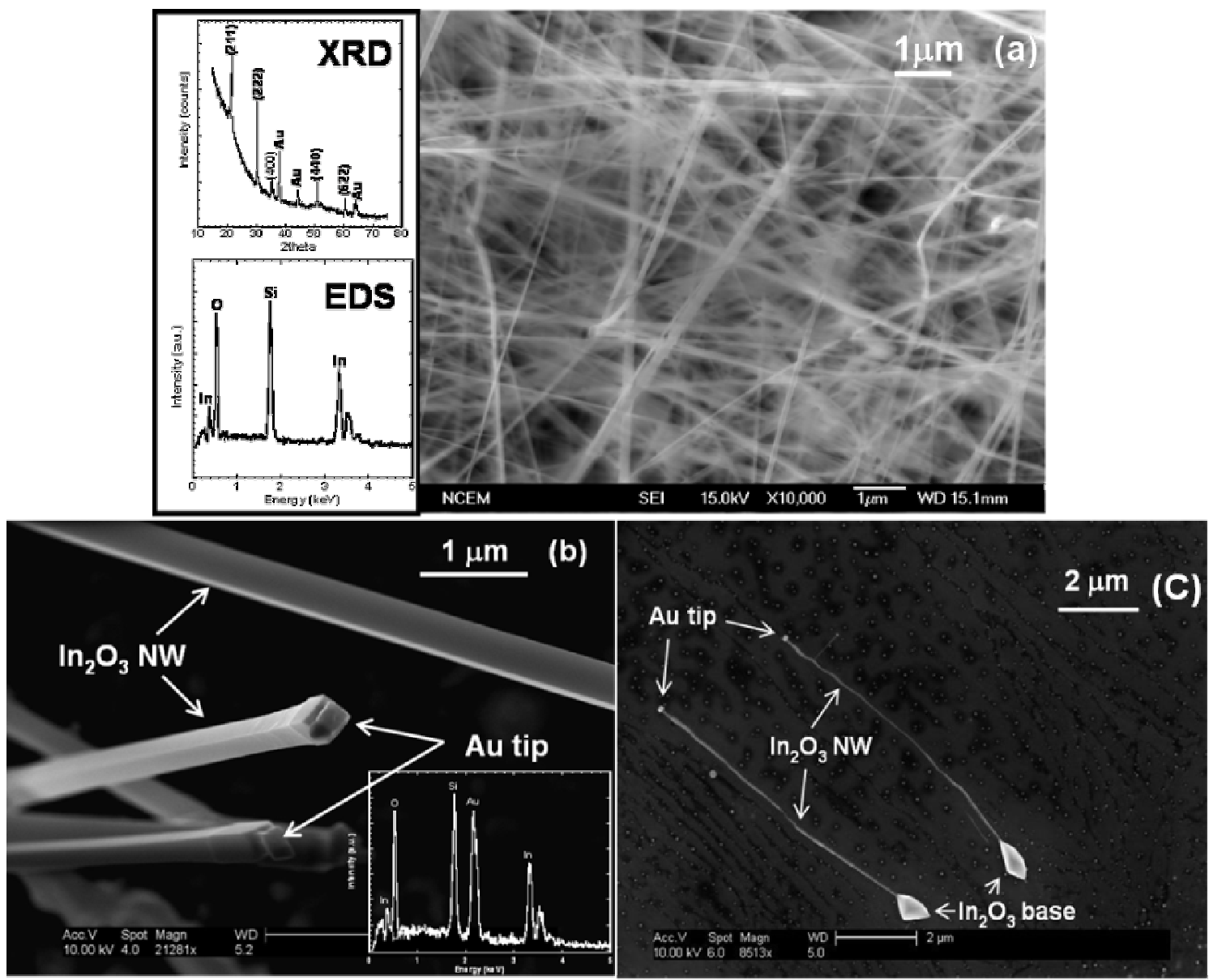
Figure 2
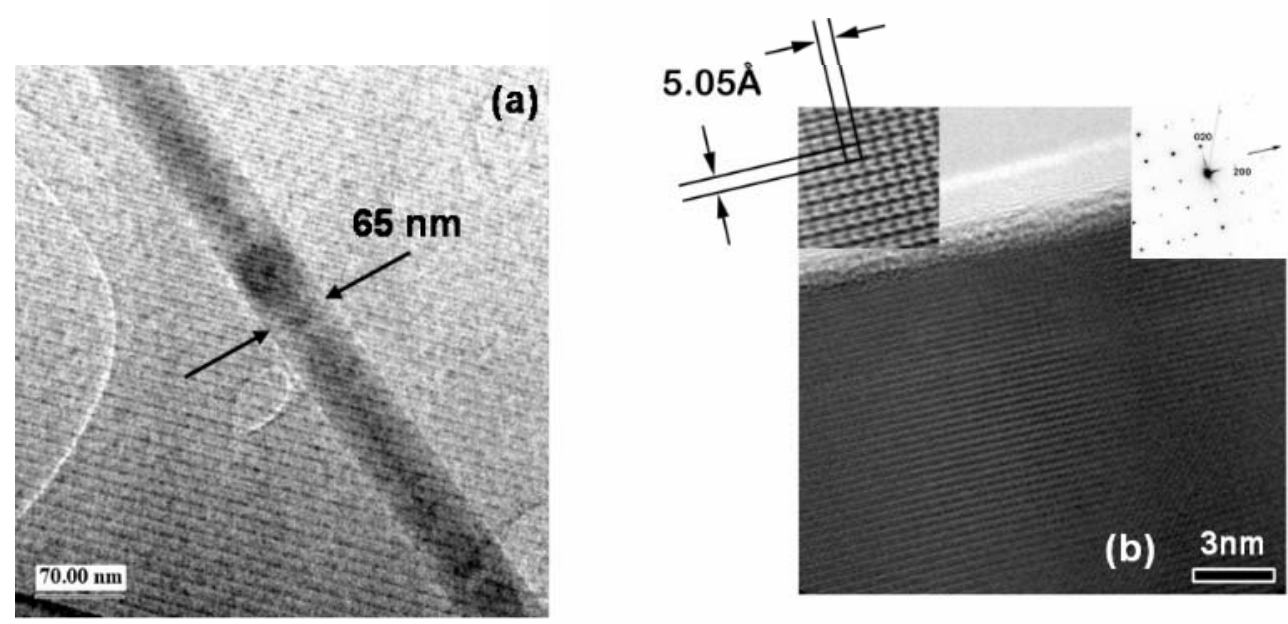
Figure 3
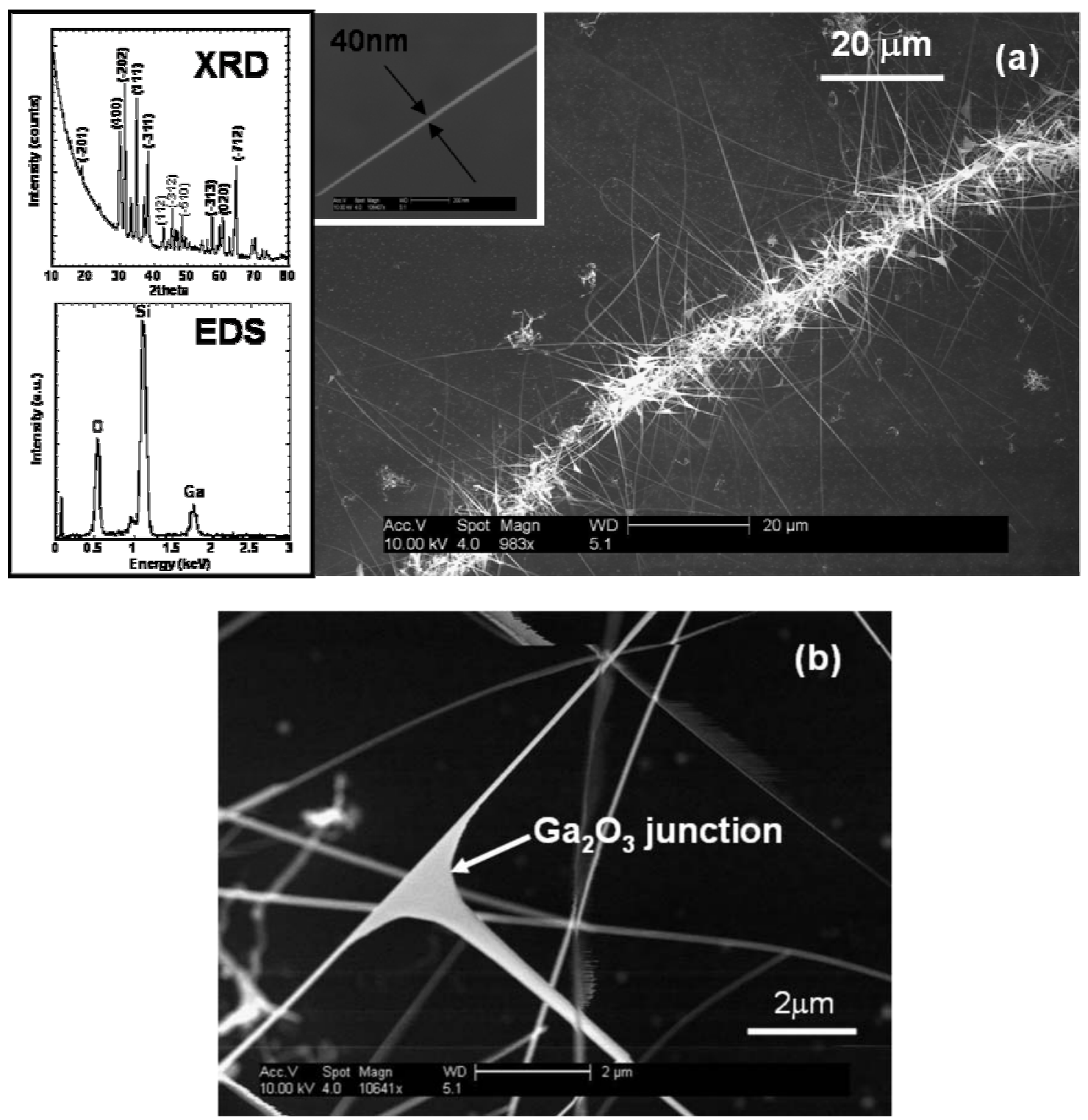
Figure 4
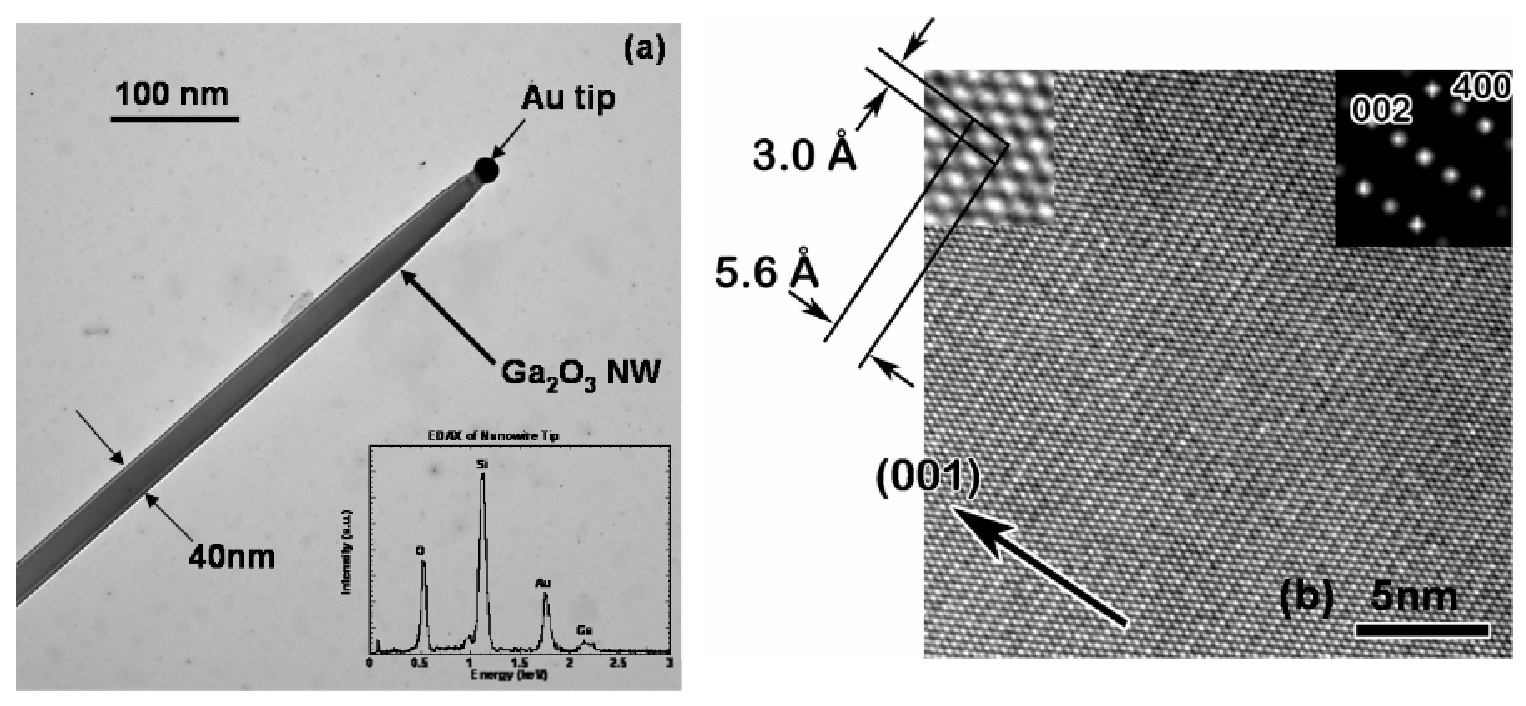
Figure 5
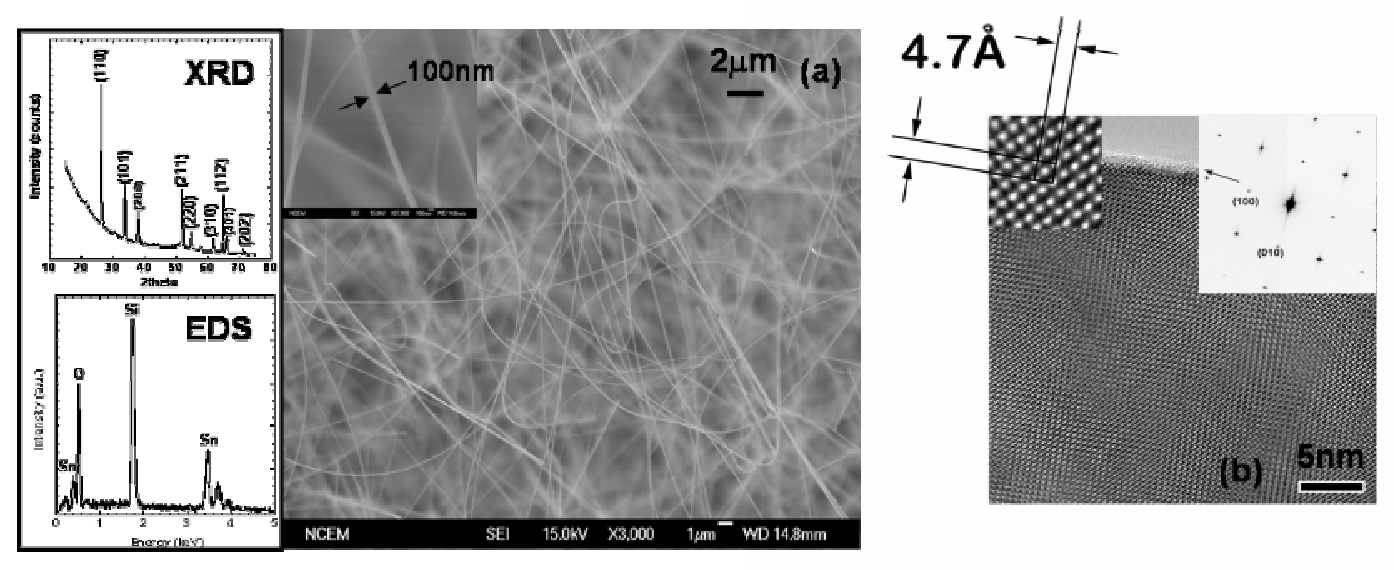
Figure 6

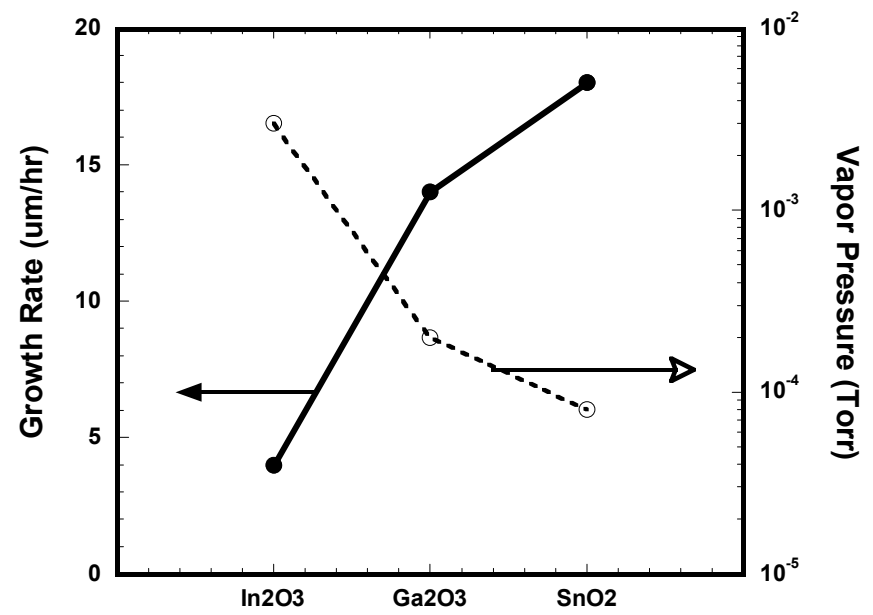

